principle of statolon and subsequently demonstrated that RNA from the mould stimulated interferon production in mice. A search for virus particles in this mould strain proved successful, whereas other, non-interferonstimulating, strains were virus free. Both the virus and RNA prepared from the virus induced production of the interferon and it was concluded that RNA (probably double stranded) of viral origin, rather than the polyanionic polysaccharide previously referred to as statolon, is the active principle in cultures of $P$. stoloniferum. Furthermore, a virus similar in morphology and size (polyhedral; 25-30 $\mathrm{nm}$ in diameter) to that found in P. stoloniferum ATCC 14586 was detected in mycelial extracts of $P$. funiculosum. These findings amply justify the earlier supposition that helenine was a double stranded RNA species of viral origin (Lampson et al., Proc. US Nat. Acad. Sci., 58, 782; 1967), and suggest promising research lines for the further study of AVS.

\section{Ion Implantation}

\section{from a Correspondent}

A RECENT two-day discussion meeting of the Royal Society held on May 2-3, and attended by over 100 participants of whom about 40 came from other European countries or the United States, was devoted to a discussion of the growing overlap of interest between hyperfine interaction measurements and the techniques of ion implantation.

The mainstream of current interest in this joint research field stems from the large magnetic hyperfine interactions experienced by a wide range of elements, including those normally diamagnetic, when present as dilute impurities in a ferromagnetic lattice. The origin of the interaction, which is of order 2-200 times stronger than can be produced by an external magnetic field, is imperfectly understood. For this reason, and because it is a valuable technique for measuring nuclear magnetic dipole moments, any extension of the range of such impurity/host systems is of interest both to nuclear and to solid state physicists.

The technique of ion implantation, the acceleration of ions to penetrate a solid lattice, has been used to extend this range in various ways. For long-lived isotopes, electro-magnetic separators are used to prepare sources implanted into ferromagnets of elements unapproachable by conventional alloying techniques through high volatility or low solubility. In addition, extremely short-lived states formed after nuclear reactions and Coulomb excitation have been made accessible to measurement by utilizing the nuclear recoil energy to displace the ion of the excited nucleus from its target lattice into a ferromagnetic foil. In this case the time taken for the recoil ion to stop is of the order $10^{-12}$ seconds.

New problems, however, arise with the use of ion implantation. The lattice position of the implanted ion and the effects of damage caused by its passage through the host lattice are both uncertain. Calculation and experiment both suggest that for the majority of soluble impurities the substitutional site is highly probable and damage effects are far enough removed from the impurity to leave the hyperfine interaction unaltered. But measurements of the Rutherford scattering of a highly collimated beam from implanted single crystals have shown a wide variation in the proportions of insoluble impurity ions occupying substitutional and interstitial sites for different impurity/ host systems and implantation conditions.

Inverting the technique and using the fact that the hyperfine interaction is sensitive to lattice site and ion charge state offers the possibility of a new method of studying the distribution of the implanted ions and their subsequent diffusion, and the "hot atom chemistry" of these ions. The method has the advantages of being sensitive to the implanted (radioactive) ions only, of being non-destructive so that a single sample may be followed through a series of-for example-thermal variations, and, by recoil implantation, being capable of measurements over an extremely wide time scale.

In addition to invited talks, contributed papers were presented on recent experiments. Measurements of the electric quadrupole interaction of hafnium displaced from its normal site in several organic complexes have shown the existence of a variety of crystalline sites and chemical species following thermal neutron irradiation. A highlight in the discussion of magnetic lattice effects was the proposal by Professor $J$. Lindhard (Aarhus) of a new mechanism-based on the close approach during scattering of the outer (partially polarized) electrons of the host ions to the impurity nucleus-for the anomalous hyperfine interactions observed in nuclear levels whose half-life is such that they decay during the stopping process. The need for further detailed investigation of the site of the implanted ion was shown by the continuing disagreement between the hyperfine interaction measured for hafnium in iron prepared respectively by conventional and implantation techniques.

\section{Iron Carriers}

\section{from our Medical Biochemistry Correspondent}

InoN in the form of haem in the cytochromes and haemoglobin is vital to mammalian life, but the way in which iron in the diet becomes incorporated into the biologically active haem proteins is still not fully understood. Iron is stored in the form of ferritin, a protein containing a large central micelle of ferric hydroxy-phosphate. It was thought that the incorporation and removal of iron from ferritin was a process which involved oxidation and reduction of the iron, but recent work suggests that this is not necessary.

One of the strongest pieces of evidence for the role of oxidation in the formation of ferritin was the observation that ferritin could not be formed from ferric salts and the protein apoferritin, but was formed when apoferritin and ferrous salts reacted in suitable oxidizing conditions. Pape, Multani, Stitt and Saltman (Biochemistry, $\%, 606 ; 1968$ ) have now shown that the "ferritin" produced by incubating apoferritin with ferrous ammonium sulphate in the presence of a stream of oxygen is different in structure from crystalline ferritin and from crystalline ferritin formed from noncrystalline ferritin and apoferritin. Electron microscopy showed that the "ferritin" from ferrous ammonium sulphate had a micelle with a diameter of only $48 \AA$, while the other ferritins had an electron dense core of diameter about $70 \AA$. The diameter of the whole molecule was about $102 \AA$ for ferrous "ferritin" and $120 \AA$ for native ferritin, indicating that the outer protein shell was the same. Non-crystalline 